\title{
Emergency Management of Acute Mastitis
}

\author{
Jinhua Ding ${ }^{1}$, Jianjiang Fang ${ }^{2}$ and Li Jiang ${ }^{2 *}$ \\ ${ }^{1}$ Department of Breast and Thyroid Surgery, China \\ ${ }^{2}$ Department of Emergency Medicine, Ningbo Medical Center Lihuili Eastern Hospital/Taipe Medical University Ningbo Medical Center, China
}

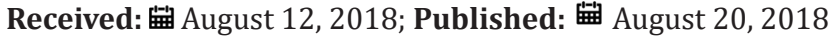

*Corresponding author: Li Jiang, Doctor of Emergency Medicine, Ningbo Medical Center Lihuili Eastern Hospital/ Taipe Medical University Ningbo Medical Center, Ningbo, China

\section{Mini Review}

Mastitis is the most common breast inflammation, and is estimated to occur in approximately one in five of postpartum women [1-3]. Mastitis is often caused by milk stasis, which has various causes, including: damaged or cracked nipples, irregular feeding, failing to empty the breast, illness in the mother or baby.

Mastitis often has a rapid progression with a manifestation of localized redness, swelling and pain in the breast, and mostly is accompanied by systemic symptoms: fever, chill, headache, nausea and vomiting. Due to the intolerable pain or high fever, patients are more likely to visit to emergency department for urgent medical help. Therefore, emergency management of acute mastitis becomes popular.

\section{Definition}

The common used definition is established by World Health Organization (WHO),as an inflammatory condition of the breast, possibly accompanied by infection and usually associated with lactation. However, inflammatory breast diseases during lactation include the followings: milk stasis, engorgement, plugged (or obstructed) ducts, and mastitis [4-6]. In addition, the treatment principle of mastitis is different to other inflammatory diseases, therefore, these diseases should be distinguished from mastitis carefully. Clinically, milk stasis, engorgement and plugged ducts have been considered as the pilot process on the formation of mastitis.

\section{Conservative Management}

Sometimes, mastitis may be non-infectious, and conservative managements are sufficient to control severe inflammation of the breast. Effective milk removal and pain medication have been the mainstays of conservative treatment. Many physical methods, such as hot or cold compress, acupuncture and microwave have been demonstrated to be inconclusive for managing milk stasis [7]. However, therapeutic breast massage has been found helpful for emptying breast and relieving milk stasis [8-10]. After one time of therapeutic breast massage named six-step recanalization manual therapy (SSRMT), as high as $98.5 \%$ milk stasis has a clinical response [9].

\section{Antibiotics Use}

In the majority of circumstance, mastitis is infectious and antibiotics use is usually based on the severity of signs and symptoms, not the bacteriological analyses, although the latter had been helpful to determine the presence of pathogenic bacteria or not. However, in the following situations:

a) Patients do not respond or are allergic to antibiotics

b) The condition recurs

c) The condition is severe or unusual, bacterial culture should be undertaken and the sensitivity tested. In spite that, there is little evidence from the randomized-controlled trials currently available to evaluate the effect of antibiotic therapy on mastitis [11]. The most common pathogen causing mastitis is Staphylococcus aureus and the antibiotics of choice include co-amoxiclav or flucloxacillin, or, for women allergic to penicillins, clarithromycin or erythromycin $[12,13]$, and these agents have been deemed safe for breastfeeding mothers.

\section{Management for Breast Abscess}

In the ultrasound imaging, there are some characteristic imaging features for breast abscess, such as multiloculated hypoechoic area, a thickened periphery and increased vascularity. Three techniques include surgical incision drainage, aspiration and ultrasound-guided drainage, have been proved to be effective methods for eliminating breast abscess. The key point of surgical incision drainage is that the incision should be large enough to ensure adequate drainage, however, it often results in a rough scar in the breast. The indication of aspiration is small-sized breast abscess, which is restricted to breast abscess no larger than $2 \mathrm{~cm}$. A recent trial demonstrated that ultrasound-guided drainage is a mini-invasive as well as effective procedure and recommended the standard treatment for abscess drainage. For those confirmed or 
suspected breast abscesses, appropriate antibiotics should also be administrated $[14,15]$. In addition, breast feeding should be encouraged during the treatment period and discontinued only when surgical incision and drainage is completed or the using antibiotics are contraindicated for new borns $[4,5]$. In the rare cases of breast abscess suspected, but ultrasound fails to demonstrate a defined fluid collection, mammography or breast biopsy should be performed to exclude the more aggressive disease, such as inflammatory carcinoma [16].

\section{Conclusion}

Acute mastitis is common in women with breast feeding, and it often shows severe inflammation with or without systemic reactions. Conservative managements, especially therapeutic breast massage, are helpful for emptying breast and relieving milk stasis. Clinically, antibiotics are often prescribed for the women with severe inflammatory signs without the result of bacterial culture.

\section{References}

1. Amir LH, Forster DA, Lumley J, McLachlan H (2014) A descriptive study of mastitis in Australian breastfeeding women: incidence and determinants. BMC Public Health 7: 62.

2. Kinlay JR, O Connell DL, Kinlay S (1998) Incidence of mastitis in breastfeeding women during the six months after delivery: a prospective cohort study. Med J Aust 169(6): 310-312.

3. Thompson JF, Roberts CL, Currie M, Ellwood DA (2002) Prevalence and persistence of health problems after childbirth: associations with parity and method of birth. Birth 29(2): 83-94.

4. Kvist LJ (2010) Toward a clarification of the concept of mastitis as used in empirical studies of breast inflammation during lactation. J Hum Lact 26(1): 53-59.

ISSN: 2574-1241

DOI: $10.26717 / B J S T R .2018 .08 .001611$

Li Jiang. Biomed J Sci \& Tech Res

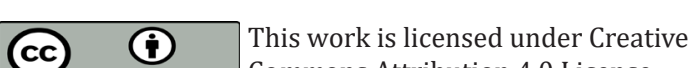

Submission Link: https://biomedres.us/submit-manuscript.php
5. Betzold CM (2007) An update on the recognition and management of lactational breast inflammation. J Midwifery Womens Health 52(6): 595605 .

6. Contreras GA, Rodriguez JM (2011) Mastitis: comparative etiology and epidemiology. J Mammary Gland Biol Neoplasia 16(4): 339-356.

7. Mangesi L, Zakarija - Grkovic I (2016) Treatments for breast engorgement during lactation. Cochrane Database Syst Rev 6: CD006946.

8. Bolman M, Saju L, Oganesyan K, Kondrashova T, Witt AM, et al. (2013) Recapturing the art of therapeutic breast massage during breastfeeding. J Hum Lact 29(3): 328-331.

9. Zhao C, Tang R, Wang J, Guan X, Zheng J, et al. (2014) Six-Step Recanalization Manual Therapy: A Novel Method for Treating Plugged Ducts in Lactating Women. J Hum Lact 30(3): 324-330.

10. Witt AM, Bolman M, Kredit S, Vanic A (2016) Therapeutic Breast Massage in Lactation for the Management of Engorgement, Plugged Ducts, and Mastitis. J Hum Lact 32(1): 123-131.

11. Jahanfar S, Ng CJ, Teng CL (2013) Antibiotics for mastitis in breastfeeding women. Cochrane Database of Systematic Reviews.

12. Balkam JJ (2010) Painful breast lumps in nursing mothers. American Journal of Nursing 110(12): 65-67.

13. Dixon JM, Khan LR (2011) Treatment of breast infection. British Medical Journal 342: 484-389.

14. Trop I, Dugas A, David J, El Khoury M, Boileau JF, et al. (2011) Breast abscesses: evidence-based algorithms for diagnosis, management, and follow-up. Radiographics 31(6): 1683-1700.

15. Leborgne F (2003) Treatment of breast abscesses with sono graphically guided aspiration, irrigation, and instillation of antibiotics. AJR Am J Roentgenol 181(4):1089-1091.

16. Berna-Serna JD, Madrigal M, Berna-Serna JD (2004) Percutaneous management of breast abscesses: an experience of 39 cases. Ultrasound Med Biol 30(1): 1-6.

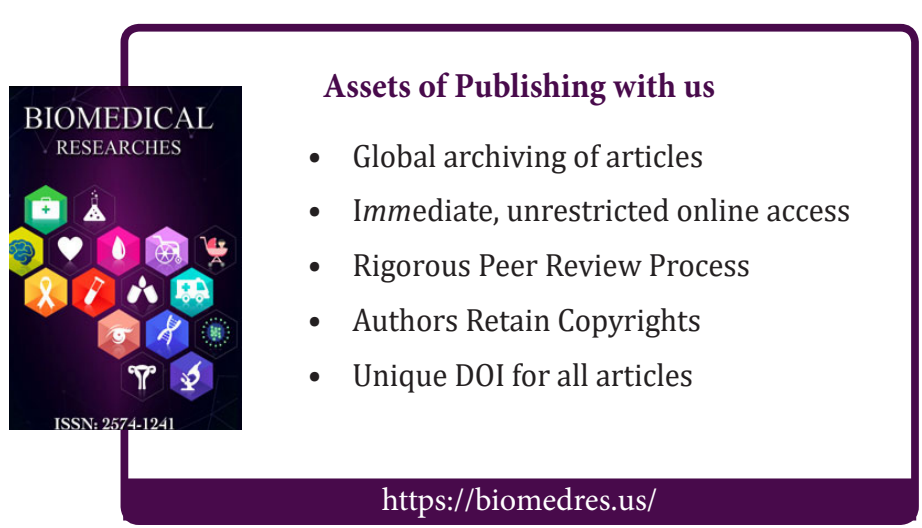

